\title{
1 Lipid-derived aldehyde degradation under thermal conditions
}

2 Rosario Zamora, José L. Navarro, Isabel Aguilar, Francisco J. Hidalgo *

3 Instituto de la Grasa, Consejo Superior de Investigaciones Científicas, Avenida Padre

4 García Tejero 4, 41012-Seville, Spain

5

6

* Corresponding author. Tel.: +34 954611 550; fax: +34 954616 790. E-mail

7 address: fhidalgo@ig.csic.es (F. J. Hidalgo)

8 


\section{ABSTRACT}

10 Nucleophile degradations produced by reactive carbonyls play a major role in food

11 quality and safety. Nevertheless, these reactions are complex because reactive carbonyls

12 are usually involved in different competitive reactions. This study describes the thermal

13 degradation of 2-alkenals (2-pentenal and 2-octenal) and 2,4-alkadienals (2,4-

14 heptadienal and 2,4-decadienal) in an attempt of both clarifying the stability of

15 aldehydes and determining new compounds that might also play a role in

16 nucleophile/aldehyde reactions. Alkenals and alkadienals decomposed rapidly in the

17 presence of buffer and air to produce formaldehyde, acetaldehyde, and the aldehydes

18 corresponding to the breakage of the carbon-carbon double bonds: propanal, hexanal, 2-

19 pentenal, 2-octenal, glyoxal, and fumaraldehyde. The activation energy of double bond

20 breakage was relatively low ( $\sim 25 \mathrm{~kJ} / \mathrm{mol})$ and the yield of alkanals (10-18\%) was higher

21 than that of 2-alkenals ( 1\%). The obtained results indicate that these reactions should

22 be considered in order to fully understand the range of nucleophile/aldehyde adducts

23 produced.

24 Keywords: Alkanals; 2,4-Alkadienals; 2-Alkenals; Aldehyde degradation; Food flavors;

25 Lipid oxidation 


\section{Introduction}

28 Reactive carbonyls are known to play a major role in some of the changes suffered by foods upon processing. These changes may have both positive and negative consequences for foods because of the formation of different compounds, including Strecker aldehydes (Maire, Rega, Cuvelier, Soto, \& Giampaoli, 2013; Rendon, Salva, \& Bragagnolo, 2014), vinylogous derivatives of amino acids such as acrylamide (Arvanitoyannis, \& Dionisopoulou, 2014; Zamora, Delgado, \& Hidalgo, 2011), biogenic amines (Granvogl, \& Schieberle, 2006; Hidalgo, Navarro, Delgado, \& Zamora, 2013), and aromatic heterocyclic amines (Zamora, Alcon, \& Hidalgo, 2012; Zochling, \& Murkovic, 2002), among others.

Reactive carbonyls are produced in foods as a consequence of oxidative and thermal processes of all major food components including carbohydrates, lipids, and amino acids or proteins (Choe, \& Min, 2006; Fuentes, Estevez, Ventanas, \& Ventanas, 2014; Zamora, \& Hidalgo, 2005; Zamora, Alcon, \& Hidalgo, 2013). Among them, lipids have long been known to be a major source of reactive carbonyls in foods (Brewer, 2009; Ganesan, Brothersen, \& McMahon, 2014).

Lipid-derived reactive carbonyls are produced in the course of lipid oxidation, and they are a large number of short- and long-chain aldehydes and ketones with various degrees of unsaturation (Gardner, 1989). Among them, 2-alkenals and 2,4-alkadienals are produced to a significant extent (Guillen and Uriarte, 2012), and they have been shown to be involved in many chemical reactions that take place in foods upon processing such as the conversion of asparagine to acrylamide (Hidalgo, Delgado, \& Zamora, 2009) or the formation of the heterocyclic aromatic amine PhIP (Zamora,

50 Alcon, \& Hidalgo, 2014), for example. These reactions require a high temperature at which unsaturated aldehydes might degrade and degradation products might also play a 
role in those reactions. However, thermal degradation of lipid-derived unsaturated

53 aldehydes is not well known, although some studies have shown that these compounds

54 are degraded upon heating (Matthews, Scanlan, \& Libbey, 1971).

In an attempt to clarify the stability of 2-alkenals and 2,4-alkadienals upon thermal processing, this study identifies and quantifies the thermal degradation products of 2alkenals and 2,4-alkadienals. As models of 2-alkenals and 2,4-alkadienals, 2-pentenal and 2,4-heptadienal, respectively, were selected as oxidation products of $\omega 3$ fatty acid chains, and 2-octenal and 2,4-decadienal, respectively, were selected as oxidation

60 products of $\omega 6$ fatty acid chains.

\section{Materials and methods}

62

63

64

\subsection{Materials}

2-Alkenals (2-pentenal and 2-octenal) and 2,4-alkadienals (2,4-heptadienal and 2,4decadienal) were purchased from Aldrich (Milwakee, WI, USA) and had the highest available grade. All other chemicals were purchased from Aldrich (Milwakee, WI, USA), Sigma (St. Louis, MO, USA), Fluka (Buchs, Switzerland), or Merck (Darmstadt, Germany), and were analytical grade.

\subsection{Thermal treatment of lipid-derived aldehydes}

Two different procedures were followed depending on whether the formed compounds were going to be either identified or quantified. The identification of thermal degradation products of the studied aldehydes was carried out by GC-MS after derivatization with $O$-(2,3,4,5,6-pentafluorobenzyl)hydroxylamine hydrochloride, according to a previously described procedure (Zamora, Navarro, Gallardo, \& Hidalgo, 2006), which was modified. Quantification of produced compounds was carried out by 
LC-MS/MS after derivatization with dansylhydrazine according to a previously described procedure (Zamora, Alcon, \& Hidalgo, 2014), which was also modified.

$$
\text { For samples with identification purposes, the aldehyde ( } 4 \mu \mathrm{mol}) \text { was either heated }
$$
alone or in the presence of $200 \mu \mathrm{L}$ of $50 \mathrm{mM}$ buffer (either sodium phosphate or sodium borate), $\mathrm{pH}$ 8, for $1 \mathrm{~h}$ at $200{ }^{\circ} \mathrm{C}$ in closed test tubes under either nitrogen or air. At the end of the heating process, samples were cooled (5 min at room temperature and $10 \mathrm{~min}$ at $\left.-20^{\circ} \mathrm{C}\right)$ and derivatizated with $400 \mu \mathrm{L}$ of a freshly prepared solution of $O-(2,3,4,5,6-$ pentafluorobenzyl)hydroxylamine hydrochloride $(10 \mathrm{mg} / \mathrm{mL}$ in methanol). The resulting solution was stirred and incubated for $1 \mathrm{~h}$ at $37^{\circ} \mathrm{C}$. After that, reactions were studied by GC-MS.

For samples with quantification purposes, a solution of the aldehyde $(0-10 \mu \mathrm{mol})$ in tetrahydrofuran $(80 \mu \mathrm{L})$ was treated with $420 \mu \mathrm{L}$ of $0.2 \mathrm{M}$ buffer (pH 2.15-11) and, then, heated for the indicated time and temperature in closed test tubes under air. At the end of the heating process, samples were cooled (5 min at room temperature and $10 \mathrm{~min}$ at $-20^{\circ} \mathrm{C}$ ). Fifty microliters of these cooled samples were diluted with $350 \mu \mathrm{L}$ of methanol, and treated with $50 \mu \mathrm{L}$ of the internal standard (a solution of $88 \mu \mathrm{mol}$ of formaldehyde- $\mathrm{d}_{2}$ in $2 \mathrm{~mL}$ of methanol), $150 \mu \mathrm{L}$ of trifluoromethanesulfonic acid solution (3\% in methanol), and $200 \mu \mathrm{L}$ of dansylhydrazine solution $(4 \mathrm{mg} / \mathrm{mL}$ in methanol). The resulting solution was incubated for $15 \mathrm{~min}$ at $100{ }^{\circ} \mathrm{C}$, then maintained for $1 \mathrm{~h}$ at $25^{\circ} \mathrm{C}$, and, finally diluted with $200 \mu \mathrm{L}$ of eluent A (a 30:70 mixture of $0.2 \%$ formic acid in acetonitrile and $4 \mathrm{mM}$ ammonium acetate), and analyzed by LC-MS/MS.

\subsection{GC-MS analyses}

GC-MS analyses were conducted with a Hewlett-Packard 6890 GC Plus coupled with an Agilent 5973 MSD (Mass Selective Detector-Quadrupole type). A fused silica 
HP5-MS capillary column $(30 \times 0.25$ mm i.d.; coating thickness $0.25 \mu \mathrm{m})$ was used.

Working conditions were as follows: carrier gas, helium (1 mL/min at constant flow); injector temperature, $250{ }^{\circ} \mathrm{C}$; oven temperature, from $70(1 \mathrm{~min})$ to $240{ }^{\circ} \mathrm{C}$ at $5{ }^{\circ} \mathrm{C} / \mathrm{min}$

102 and, then, to $325^{\circ} \mathrm{C}$ at $10{ }^{\circ} \mathrm{C} / \mathrm{min}$; transfer line to MSD, $280{ }^{\circ} \mathrm{C}$; ionization EI, $70 \mathrm{eV}$.

103 Reaction products were identified by comparison of mass spectra and retention times of 104 those of authentic standards.

\subsection{LC-MS/MS analyses}

The employed equipment was composed by an Agilent liquid chromatography system (1200 Series) consisting of binary pump (G1312A), degasser (G1379B), and autosampler (G1329A), connected to a triple quadrupole API 2000 mass spectrometer

109 (Applied Biosystems, Foster City, CA) using an electrospray ionization interface in 110 positive ionization mode $\left(\mathrm{ESI}^{+}\right)$. Compounds were separated on a Zorbax Eclipse XDB-

111 C18 (150 mm x $4.6 \mathrm{~mm}, 5 \mu \mathrm{m})$ column from Agilent. As eluent A, a 30:70 mixture of

$1120.2 \%$ formic acid in acetonitrile and $4 \mathrm{mM}$ ammonium acetate was used. As eluent $\mathrm{B}$, a

$1130.2 \%$ formic acid solution in acetonitrile was employed. The mobile phase was

114 delivered at $0.5 \mathrm{~mL} / \mathrm{min}$ using the following gradient: for $0-13 \mathrm{~min}$, the content of

115 mobile phase B was 7\%; for 13-20 min, the content of mobile phase B was increased

116 linearly from 7 to $60 \%$; for $20-30 \mathrm{~min}$, the content of mobile phase B was $60 \%$; for 30

$11732 \mathrm{~min}$, the content of mobile phase B was increased linearly from 60 to $90 \%$; for 32-42

118 min, the content of mobile phase B was $90 \%$; and for $42-45$ min, the content of mobile

119 phase B was decreased linearly from 90 to 7\%. Mass spectrometric acquisition was

120 performed by using multiple reaction monitoring (MRM). The nebulizer gas (synthetic

121 air), the curtain gas (nitrogen), and the heater gas (synthetic air) were set at 40, 25, and

12250 (arbitrary units), respectively. The collision gas (nitrogen) was set at 3 (arbitrary

123 units). The heater gas temperature was set at $500{ }^{\circ} \mathrm{C}$ and the electrospray capillary 
124 voltage to $5.5 \mathrm{kV}$. The fragment ions in MRM mode were produced by collision-

125 activated dissociation of selected precursor ions in the collision cell of the triple

126 quadrupole and the selected products analyzed with the second analyzer of the

127 instrument. Three transitions were acquired for the identification of each

128 dansylhydrazone derivative. To establish the appropriate MRM conditions for the

129 individual compounds, the mass spectrometric conditions were optimized using infusion

130 with a syringe pump to select the most suitable ion transitions for the target analytes.

131 Precursor and product ions used for quantification and confirmation purposes, and

132 operating conditions are summarized in Table 1.

133 Quantification of the different aldehydes was carried out by preparing five standard

134 curves of aldehyde mixtures in $500 \mu \mathrm{L}$ of the mixture tetrahydrofuran/sodium phosphate

135 buffer, $\mathrm{pH}$ 8, and following the whole procedure described above. For each curve, seven

136 different concentration levels of aldehydes (0-2 nmol) were used. Aldehyde contents

137 were directly proportional to aldehyde/IS area ratios $(\mathrm{r}>0.99, p<0.0001)$. All data

138 given are mean of, at least, three independent experiments.

\section{3. Results}

140 3.1. Thermal degradation of 2-alkenals and 2,4-alkadienals

$141 \quad$ 2-Alkenals and 2,4-alkadienals were more or less stable upon heating depending on

142 the presence of both air and buffers. Fig. 1 shows the chromatograms obtained after $1 \mathrm{~h}$

143 heating at $200{ }^{\circ} \mathrm{C}$ for the four assayed aldehydes: 2-pentenal (chromatograms a-d), 2-

144 octenal (chromatograms e-h), 2,4-heptadienal (chromatograms i-l), and 2,4-decadienal

145 (chromatograms m-p). The first chromatogram of each series (chromatograms a, e, i,

146 and m) corresponded to the aldehyde with not solvent added and heated under nitrogen.

147 The second chromatogram of each series (chromatograms b, $\mathrm{f}, \mathrm{j}$, and $\mathrm{n}$ ) corresponded to 
148 the aldehyde with not solvent added and heated under air. The third chromatogram of

149 each series (chromatograms c, g, k, and o) corresponded to the solution of the aldehyde

150 in a buffer which was heated under nitrogen. The fourth chromatogram of each series

151 (chromatograms d, h, l, and p) corresponded to the solution of the aldehyde in a buffer

152 which was heated under air. Because the results obtained using either sodium phosphate

153 buffer, $\mathrm{pH} \mathrm{8,} \mathrm{or} \mathrm{sodium} \mathrm{borate} \mathrm{buffer,} \mathrm{pH}$ 8, were identical, only the chromatograms

154 obtained using sodium phosphate buffer are shown (chromatograms c, d, g, h, k, l, o,

155 and $\mathrm{p})$.

156 2-Alkenals and 2,4-alkadienals mostly remained unchanged in the absence of both

157 buffer and air. Thus, 2-pentenal (4) with not solvent added was relatively stable after

158 heating under nitrogen (chromatogram a). Something similar occurred for 2-octenal (7,

159 chromatogram e), 2,4-heptadienal (6, chromatogram i), and 2,4-decadienal (9,

160 chromatogram m).

161 The most significant change produced when the aldehyde with not solvent added was

162 heated in the presence of air was the appearance of formaldehyde (1, chromatograms b,

$163 \mathrm{f}$, j, and n, for the heating of 2-pentenal, 2-octenal, 2,4-heptadienal, and 2,4-decadienal,

164 respectively, under air). In addition, the formation of minute amounts of glyoxal (8) and

165 fumaraldehyde (10) were also observed in chromatograms j and n. Furthermore, the

166 formation of trace amounts of 2-pentenal (4) and propanal (3) in chromatogram j, and of

167 2-octenal (7) and hexanal (5) in chromatogram n were also observed.

168 Aldehydes suffered a higher decomposition in the present of buffer. However,

169 dialdehydes $\mathbf{8}$ and $\mathbf{1 0}$ were not observed under these reaction conditions, and

170 formaldehyde (1) was detected to a lower extent than when the buffer was absent. On

171 the other hand, shorter aldehydes were produced to a higher extent and a similar

172 decomposition was observed in the presence and in the absence of air. Thus, 2-pentenal 
173 (4) disappeared completely after $1 \mathrm{~h}$ at $200{ }^{\circ} \mathrm{C}$ (chromatograms $\mathrm{c}$ and $\mathrm{d}$ for nitrogen and

174 air, respectively) and the formation of propanal (3) and acetaldehyde (2) was observed.

175 Something similar occurred for 2-octenal (7). It almost disappeared and the formation of

176 hexanal (5) and acetaldehyde (2) was observed (chromatograms g and h for nitrogen

177 and air, respectively). 2,4-Heptadienal (6) resulted slightly more stable and the initial

178 aldehyde could still be detected after $1 \mathrm{~h}$ heating at $200^{\circ} \mathrm{C}$ (chromatograms $\mathrm{k}$ and $\mathrm{l}$ for

179 nitrogen and air, respectively). In addition, 2,4-heptadienal decomposition produced

180 propanal (3), acetaldehyde (2) and small amounts of 2-pentenal (4). Finally,

181 decomposition of 2,4-decadienal (9) (chromatograms o and p for nitrogen and air,

182 respectively) mostly produced hexanal (5) and acetaldehyde (2).

183 With the exception of formaldehyde and acetaldehyde, the formed aldehydes

184 corresponded to the breakage of the different double bonds present in the initial

185 aldehyde as indicated in Fig. 1. Thus, 2-alkenals ( $\mathbf{4}$ or $\mathbf{7}$ ) produced the corresponding

186 alkanals 3 or $\mathbf{5}$. In addition, 2,4-alkadienals (6 or $\mathbf{9}$ ) produced both 2-alkenals (4 or 7,

187 respectively) and alkanals (3 or 5, respectively). These reactions were accompanied

188 with the formation of both glyoxal (8) and fumaraldehyde (10), although these last

189 compounds seemed to be easily decomposed when buffer was present. Next sections

190 will describe the formation of shorter aldehydes by thermal breakage of carbon-carbon

191 double bonds in 2-alkenals and 2,4-alkadienals.

192

\subsection{Thermal degradation of 2-pentenal}

193

As discussed previously, the breakage of 2-pentenal produced propanal, in addition

194 to formaldehyde and acetaldehyde. This reaction should be accompanied by the

195 formation of glyoxal, although this compound was not detected when the reaction was

196 carried out in the presence of buffer. 2-Pentenal decomposition in buffer solution and

197 the formation of the corresponding propanal is shown in Fig. 2. As can be observed, 
propanal was formed to an extent that depended on the concentration of 2-pentenal and

199 the reaction conditions. Fig. 2A shows that propanal formation took place over a wide

200 pH-range with a maximum around $\mathrm{pH}$ 8. The amount of produced propanal increased

201 linearly ( $\mathrm{r}=0.993, p<0.0001)$ as a function of 2-pentenal concentration (Fig. 2B). The

202 slope of the obtained line (0.125) indicated the reaction yield (12.5\%), which was

203 constant over the assayed concentration range (0-80 $\mu \mathrm{mol}$ of 2-pentenal).

204 2-Pentenal concentration decreased exponentially as a function of heating time and

205

206

207

208

209

210

211

212

213 temperature (Fig. 2C), and 2-pentenal disappearance was more rapidly produced at a

higher temperature. Thus, less than $10 \%$ of initial pentanal was observed after 25 min at $200^{\circ} \mathrm{C}$ and after $45 \mathrm{~min}$ at $160{ }^{\circ} \mathrm{C}$. When 2-pentenal was heated at $120^{\circ} \mathrm{C}, 17 \%$ of the initial aldehyde was still present after $60 \mathrm{~min}$.

This disappearance of 2-pentenal was parallel to the formation of propanal (Fig. 2D). Propanal concentration only increased linearly $(r>0.994, p<0.0067)$ for most temperatures at the beginning of the heating, in accordance to the exponential degradation observed for 2-pentenal. In fact, there was an inverse correlation $(r>0.935$, $p<0.002$ ) between the concentrations of 2-pentenal and propanal as a function of heating time at the three assayed temperatures.

Reaction rates for propanal formation were higher at higher temperatures. These reaction rates were calculated from the initial times in which the concentration of propanal increased linearly as a function of heating time (Fig. 2D) by using the equation

$$
\text { [propanal] }=k t
$$

where $\mathrm{k}$ is the rate constant and $t$ is the time. These rate constants were used in an Arrhenius plot for the calculation of the activation energy $\left(E_{a}\right)$ of propanal formation by heating 2-pentenal. The determined $E_{\mathrm{a}}$ was $25.2 \mathrm{~kJ} / \mathrm{mol}$. 


\subsection{Thermal degradation of 2-octenal}

223

2-Octenal exhibited a behavior analogous to that of 2-pentenal, and hexanal

224

formation also depended on 2-octenal concentration and reaction conditions (Fig. 3).

225

Hexanal was mainly produced at basic $\mathrm{pH}$, with a maximum around $\mathrm{pH} 10$ (Fig. 3A).

226

The amount of hexanal formed increased linearly ( $r=0.999, p<0.0001)$ as a function of 2-octenal concentration (Fig. 3B). The slope of the obtained line (0.180) indicated the reaction yield (18.0\%) which was constant over the assayed range (0-80 $\mu \mathrm{mol}$ of 2 octenal). This yield was slightly higher than that found for the formation of propanal from 2-pentenal.

Analogously to 2-pentenal, 2-octenal also disappeared exponentially as a function of reaction time and this disappearance was produced more rapidly at a higher temperature (Fig. 3C). Less than $10 \%$ of the initial 2-octenal was found after 10 min heating at 200 ${ }^{\circ} \mathrm{C}$, 50 min heating at $160{ }^{\circ} \mathrm{C}$, and about 60 min when heating at $120^{\circ} \mathrm{C}$.

Hexanal concentration increased linearly $(r>0.971, p<0.00097)$ as a function of reaction time, and reaction rates were higher at higher temperatures (Fig. 3D). Reaction rates were calculated from the slopes of the adjusted lines as described previously. The determined $E_{\text {a }}$ was $25.3 \mathrm{~kJ} / \mathrm{mol}$, which was very similar to the $E_{\text {a }}$ obtained for propanal formation from 2-pentenal (see above).

\subsection{Thermal degradation of 2,4-heptadienal}

When 2,4-heptadienal was heated in the presence of buffer, the formation of the two aldehydes corresponding to the breakage of either one or the other double bond was

243 observed, although propanal was always formed to a higher extent than 2-pentenal (Fig.

244 4). In addition, and analogously to 2-pentenal and 2-octenal decomposition, aldehyde formation depended on the concentration of 2,4-heptadienal and the reaction conditions. 
Propanal and 2-pentenal were produced over a wide $\mathrm{pH}$ range with a maximum at about $\mathrm{pH}$ 7-8. There was not a clear difference between the optimum $\mathrm{pH}$ values for the formation of both propanal and 2-pentenal, although 2-pentenal seemed to be produced better at a pH slightly more acidic than propanal (Fig. 4A). (Fig. 4B). This increase was lineal $(r=0.995, p<0.0001)$ for 2-pentenal for the whole concentration range assayed ( $0-80 \mu \mathrm{mol}$ of 2,4-heptadienal), and also for propanal $(\mathrm{r}=$ 0.996, $p<0.0001$ ) but only in the $0-40 \mu \mathrm{mol}$ range of 2,4-heptadienal. The slopes of the obtained lines (0.09774 and 0.00973$)$ indicated reaction yields of $9.8 \%$ and $1.0 \%$ for propanal and 2-pentenal, respectively.

Analogously to the above discussed behavior of 2-alkenals, 2,4-heptadienal was higher at higher temperature (Fig. 4C). This decrease was parallel to the formation of both propanal (Fig. 4D) and 2-pentenal (Fig. 4E). The Ea required for the formation of both aldehydes was calculated by using the slopes of the obtained lines as described previously. The $E_{\mathrm{a}}$ for propanal and 2-pentenal formation were 25.2 and $22.5 \mathrm{~kJ} / \mathrm{mol}$, respectively.

\subsection{Thermal degradation of 2,4-decadienal}

Analogously to the above described for 2,4-heptadienal, when 2,4-decadienal was heated, the formation of the two aldehydes corresponding to the breakage of the two double bonds was observed and hexanal was always formed to a higher extent than 2octenal (Fig. 5). In addition, and analogously to the above described decompositions for the other aldehydes, the yields of hexanal and 2-octenal formation depended on the concentration of 2,4-decadienal and the reaction conditions. 
Hexanal and 2-octenal were produced over a wide $\mathrm{pH}$ range with a maximum at

271 about $\mathrm{pH} 8$ and there was not a clear difference between the optimum $\mathrm{pH}$ values for the

272 formation of both aldehydes. Nevertheless, and in accordance with the observed above

273 for 2,4-heptadienal decomposition, the 2-alkenal seemed to be produced better at a pH

274 value slightly more acidic than the alkanal (Fig. 5A).

275 Hexanal and 2-octenal increased as a function of 2,4-decadienal concentration (Fig.

276 5B). This increase was lineal $(r>0.998, p<0.0001)$ for both hexanal and 2-octenal for

277 the whole concentration range assayed ( $0-80 \mu \mathrm{mol}$ of 2,4-decadienal). The slopes of the

278 obtained lines ( 0.1154 and 0.00821$)$ indicated reaction yields of $11.5 \%$ and $0.8 \%$ for

279 hexanal and 2-octenal, respectively.

280 As observed for other aldehydes, 2,4-decadienal concentration decreased

exponentially as a function of reaction time and this decrease was higher at higher

282

temperature (Fig. 5C). This decrease was parallel to the formation of both hexanal (Fig.

5D) and 2-octenal (Fig. 5E). The $E_{\text {a }}$ required for the formation of both aldehydes was

284 calculated by using the slopes of the obtained lines as described previously. The $E_{\mathrm{a}}$ for

285 hexanal and 2-octenal formation were 21.3 and $29.6 \mathrm{~kJ} / \mathrm{mol}$, respectively.

\section{4. Discussion}

Lipid oxidation is a complex cascade of reactions in which primary, secondary and tertiary lipid oxidation products are produced (Bekhit, Hopkins, Fahri, \& Ponnampalam, 2013; Ibargoitia, Sopelana, \& Guillen, 2014; Maqsood, Benjakul, \& Kamal-Eldin, 2012; Varlet, Prost, \& Serot, 2007). Some of these compounds are stable, such as alkanes. However, other lipid oxidation products are unstable and are usually involved in further reactions, which might also imply other food components. Among them, aldehydes can be either oxidized to the corresponding acids or reduced to alcohols by 
both chemical and enzymatic processes. In addition, and as described in this study, unsaturated aldehydes can also be degraded as a consequence of thermal heating.

According to the above results, the stability of the aldehydes depended on the presence of buffer and oxygen. Aldehydes resulted to be relatively stable in the absence of buffer and oxygen, but when aqueous solutions were employed, a rapid decomposition was observed. This decomposition was similar for 2-alkenals and 2,4alkadienals and always produced shorter aldehydes, among other compounds. The aldehydes produced were formaldehyde, acetaldehyde and the corresponding carbonyl compounds produced as a consequence of the breakage of the carbon-carbon double bonds present in the molecule. Thus, because 2-alkenals only have one carbon-carbon double bond, the products formed were alkanals and glyoxal. The reaction was more complex for 2,4-alkadienals because these compounds have two carbon-carbon double bonds. The breakage of the double bond between C2 and C3 produced 2-alkenals and glyoxal, and the breakage of the double bond between C4 and C5 produced alkanals and fumaraldehyde.

The $E_{\text {a }}$ for the breakage of the different carbon-carbon double bonds was always very

310 similar and was about $25 \mathrm{~kJ} / \mathrm{mol}$. However, alkanals were produced to a much higher 311 extent than 2-alkenals. Thus, $10-18 \%$ of the initial either 2-alkenal or 2,4-alkadienal was converted into alkanal after $1 \mathrm{~h}$ heating at $200{ }^{\circ} \mathrm{C}$ and only about $1 \%$ of the initial 2,4-alkadienal was converted into 2-alkenal under the same reaction conditions. The

314 lower amount of 2-alkenals found during 2,4-alkadienal degradation in relation to that 315 of alkanals is likely a consequence of the degradation suffered by 2-alkenals, which also 316 produce alkanals. However, alkanals were also produced directly from 2,4-alkadienals because fumaraldehyde was found in these reactions (Figs. $1 \mathrm{j}$ and $1 \mathrm{n}$ for 2,4-heptadienal and 2,4-decadienal, respectively). 

both their contribution to food aroma and their ability to induce changes in surrounding food components. Thus, once produced, they are further involved in reactions with the nucleophiles present in food products (Choe \& Min, 2006; Hidalgo \& Zamora, 2014; Hidalgo \& Zamora, in press; Tang, Wang, Hu, Chen, Akao, Feng, \& Hu, 2011). In

324 addition, the results obtained in the present study show that unsaturated lipid-derived 325 aldehydes are degraded. Therefore, these degradations should also be considered to fully understand the range of nucleophile/aldehyde adducts formed as well as the changes produced in the volatile composition of foods during processing or storage, and the role of aldehyde degradation products in the produced food changes. Thus, for example, in a recent study Lee and Pangloli (2013) analyzed the changes of volatile compounds produced during the storage of potato chips fried in mid-oleic sunflower oil. They found that the concentration of hexanal increased upon storage at the same time that the concentration of decadienal seemed to decrease slightly, which is in agreement with the results obtained in the present study. Moreover, polymers formed by reaction

334 between amino acids and alkadienals have been traditionally believed to be produced

335 between the amino acid and the aldehyde (see, for example, Adams, Kitryte,

336 Venskutonis \& De Kimpe, 2009). However, the results obtained in the present study suggest a potential role in these reactions of the dicarbonyl compounds (glyoxal and fumaraldehyde) produced by alkadienal decomposition.

\section{Acknowledgments}

340 This study was supported in part by the European Union (FEDER funds) and the

341 Plan Nacional de I + D of the Ministerio de Economía y Competitividad of Spain (project AGL2012-35627). 


\section{References}

344 Adams, A., Kitryte, V., Venskutonis, R., \& De Kimpe, N. (2009). Formation and

345 characterisation of melanoidin-like polycondensation products from amino acids and 346 lipid oxidation products. Food Chemistry, 115, 904-911.

347 Arvanitoyannis, I. S., \& Dionisopoulou, N. (2014). Acrylamide: Formation, occurrence

348 in food products, detection methods, and legislation. Critical Reviews in Food

$349 \quad$ Science and Nutrition, 54, 708-733.

350 Bekhit, A. E. A., Hopkins, D. L., Fahri, F. T., \& Ponnampalam, E. N. (2013). Oxidative

351 processes in muscle systems and fresh meat: sources, markers, and remedies.

352 Comprehensive Reviews in Food Science and Food Technology, 12, 565-597.

353 Brewer, M. S. (2009). Irradiation effects on meat flavor. Meat Science, 81, 1-14.

354 Choe, E., \& Min, D. B. (2006). Chemistry and reactions of reactive oxygen species in

355 foods. Critical Reviews in Food Science and Nutrition, 46, 1-22.

356 Fuentes, V., Estevez, M., Ventanas, J., \& Ventanas, S. (2014). Impact of lipid content

357 and composition on lipid oxidation and protein carbonylation in experimental

358 fermented sausages. Food Chemistry, 147, 70-77.

359 Ganesan, B., Brothersen, C., \& McMahon, D. J. (2014). Fortification of foods with

360 omega-3 polyunsaturated fatty acids. Critical Reviews in Food Science and

$361 \quad$ Nutrition, 54, 98-114.

362 Gardner, H. W. (1989). Oxygen radical chemistry of polyunsaturated fatty acids. Free

363 Radical Biology and Medicine, 7, 65-86. 
364 Granvogl, M., \& Schieberle, P. (2006). Thermally generated 3-aminopropionamide as a

365 transient intermediate in the formation of acrylamide. Journal of Agricultural and

$366 \quad$ Food Chemistry, 54, 5933-5938.

367 Guillen, M. D., \& Uriarte, P. S. (2012). Study by 1H NMR spectroscopy of the

368 evolution of extra virgin olive oil composition submitted to frying temperature in an

369 industrial fryer for a prolonged period time. Food Chemistry, 2012, 162-172.

370 Hidalgo, F. J., Delgado, R. M., \& Zamora, R. (2009). Degradation of asparagine to

371 acrylamide by carbonyl-amine reactions initiated by alkadienals. Food Chemistry, $372 \quad 116,779-784$.

373 Hidalgo, F. J., Navarro, J. L., Delgado, R. M., \& Zamora, R. (2013). Histamine

374 formation by lipid oxidation products. Food Research International, 52, 206-213.

375 Hidalgo, F. J., \& Zamora, R. (2014). 2-Alkenal-scavenging ability of m-diphenols.

$376 \quad$ Food Chemistry, 160, 118-126.

377 Hidalgo, F. J., \& Zamora, R. (in press). Amino acid degradations produced by lipid 378 oxidation products. Critical Reviews in Food Science and Nutrition, in press. DOI: $379 \quad 10.1080 / 10408398.2012 .761173$.

380 Ibargoitia, M. L., Sopelana, P., \& Guillen, M. D. (2014). (1)H Nuclear Magnetic

381 Resonance monitoring of the degradation of margarines of varied compositions when 382 heated to high temperature. Food Chemistry, 165, 119-128.

383 Lee, J. H., \& Pangloli, P. (2013). Volatile compounds and storage stability of potato 384 chips fried in mid-oleic sunflower oil. International Journal of Food Properties, 16, $385 \quad 563-573$. 
Maire, M., Rega, B., Cuvelier, M. E., Soto, P., \& Giampaoli, P. (2013). Lipid oxidation in baked products: Impact of formula and process on the generation of volatile compounds. Food Chemistry, 141, 3510-3518.

Maqsood, S., Benjakul, S., \& Kamal-Eldin, A. (2012). Haemoglobin-mediated lipid oxidation in the fish muscle: A review. Trends in Food Science \& Tecnology, 2012, 33-43.

Matthews, R. F., Scanlan, R. A., \& Libbey, L. M. (1971). Autoxidation products of 2,4decadienal. Journal of the American Oil Chemists’ Society, 48, 745-747.

Rendon, M. Y., Salva, T. D. G., \& Bragagnolo, N. (2014). Impact of chemical changes on the sensory characteristics of coffee beans during storage. Food Chemistry, 147, 279-286.

Tang, M. S., Wang, H. T., Hu, Y., Chen, W. S., Akao, M., Feng, Z. H., \& Hu, W. W. (2011). Acrolein induced DNA damage, mutagenicity and effect on DNA repair. Molecular Nutrition \& Food Research, 55, 1291-1300.

400

401

Varlet, V., Prost, C., \& Serot, T. (2007). Volatile aldehydes in smoked fish: Analysis 402 methods, occurrence and mechanisms of formation. Food Chemistry, 105, 1536-

Zamora, R., Alcon, E., \& Hidalgo, F. J. (2012). Effect of lipid oxidation products on the formation of 2-amino-1-methyl-6-phenylimidazo[4,5-b]pyridine (PhIP) in model systems. Food Chemistry, 135, 2569-2574.

406

Zamora, R., Alcon, E., \& Hidalgo, F. J. (2013). Effect of amino acids on the formation of 2-amino-1-methyl-6-phenylimidazo[4,5-b]pyridine (PhIP) in mixtures. Food Chemistry, 141, 4240-4245. 
410 Zamora, R., Alcon, E., \& Hidalgo, F. J. (2014). Ammonia and formaldehyde participate

411 in the formation of 2-amino-1-methyl-6-phenylimidazo[4,5-b]pyridine (PhIP) in

412 addition to creati(ni)ne and phenylacetaldehyde. Food Chemistry, 155, 74-80.

413 Zamora, R., Delgado, R. M., \& Hidalgo, F. J. (2011). Strecker aldehydes and $\alpha$-keto

414 acids, produced by carbonyl-amine reactions, contribute to the formation of

415 acrylamide. Food Chemistry, 128, 465-470.

416 Zamora, R., Navarro, J. L., Gallardo, E., \& Hidalgo, F. J. (2006). Chemical conversion

417 of $\alpha$-amino acids into $\alpha$-keto acids by 4,5-epoxy-2-decenal. Journal of Agricultural

$418 \quad$ and Food Chemistry, 54, 6101-6105.

419 Zamora, R., \& Hidalgo, F. J. (2005). Coordinate contribution of lipìd oxidation and

420 Maillard reaction to the nonenzymatic food browning. Critical Reviews in Food

$421 \quad$ Science and Nutrition, 45, 49-59.

422 Zochling, S., \& Murkovic, M. (2002). Formation of the heterocyclic aromatic amine

423 PhIP: Identification of precursors and intermediates. Food Chemistry, 79, 125-134. 


\section{Figure legends}

Fig. 1. Total ion chromatograms obtained for: a, 2-pentenal heated under nitrogen; b, 2pentenal heated under air; c, a solution of 2-pentenal in sodium phosphate buffer heated under nitrogen; d, a solution of 2-pentenal in sodium phosphate buffer heated under air; e, 2-octenal heated under nitrogen; f, 2-octenal heated under air; g, a solution of 2octenal in sodium phosphate buffer heated under nitrogen; h, a solution of 2-octenal in sodium phosphate buffer heated under air; i, 2,4-heptadienal heated under nitrogen; j, 2,4-heptadienal heated under air; k, a solution of 2,4-heptadienal in sodium phosphate buffer heated under nitrogen; 1, a solution of 2,4-heptadienal in sodium phosphate buffer heated under air; m, 2,4-decadienal heated under nitrogen; n, 2,4-decadienal heated under air; o, a solution of 2,4-decadienal in sodium phosphate buffer heated under nitrogen; p, a solution of 2,4-decadienal in sodium phosphate buffer heated under air. All samples were heated for $1 \mathrm{~h}$ at $200{ }^{\circ} \mathrm{C}$ and, then, derivatizated with $O-(2,3,4,5,6-$ pentafluorobenzyl)hydroxylamine hydrochloride. Compounds identified were: formaldehyde (1), acetaldehyde (2), propanal (3), 2-pentenal (4), hexanal (5), 2,4heptadienal (6), 2-octenal (7), glyoxal (8), 2,4-decadienal (9), and fumaraldehyde (10).

Fig. 2. Formation of propanal by thermal decomposition of 2-pentenal: A, effect of pH in the formation of propanal; B, effect of 2-pentenal concentration in the formation of propanal; C, time-course of 2-pentenal disappearance; and D, time-course of propanal formation. Reactions were heated at $200(\bigcirc), 160(\triangle)$, or $120^{\circ} \mathrm{C}(\nabla)$ for $1 \mathrm{~h}$ in panels $\mathrm{A}$ and $\mathrm{B}$, and the indicated times in panels $\mathrm{C}$ and $\mathrm{D}$.

Fig. 3. Formation of hexanal by thermal decomposition of 2-octenal: A, effect of $\mathrm{pH}$ in the formation of hexanal; B, effect of 2-octenal concentration in the formation of hexanal; C, time-course of 2-octenal disappearance; and D, time-course of hexanal 
formation. Reactions were heated at $200(\bigcirc), 160(\triangle)$, or $120^{\circ} \mathrm{C}(\nabla)$ for $1 \mathrm{~h}$ in panels $\mathrm{A}$ and $\mathrm{B}$, and the indicated times in panels $\mathrm{C}$ and $\mathrm{D}$.

Fig. 4. Formation of propanal (open symbols) and 2-pentenal (closed symbols) by thermal decomposition of 2,4-heptadienal: A, effect of $\mathrm{pH}$ in the formation of propanal (O) and 2-pentenal ( $\bigcirc$ ); B, effect of 2,4-heptadienal concentration in the formation of propanal $(\bigcirc)$ and 2-pentenal $(\mathbf{O})$; C, time-course of 2,4-heptadienal disappearance; D, time-course of propanal formation; and E, time-course of 2-pentenal formation.

Reactions were heated at $200(\bigcirc, \boldsymbol{\bullet}), 160(\triangle, \boldsymbol{\Delta})$, or $120^{\circ} \mathrm{C}(\nabla, \boldsymbol{\nabla})$ for $1 \mathrm{~h}$ in panels A and $\mathrm{B}$, and the indicated times in panels $\mathrm{C}$, D, and $\mathrm{E}$.

Fig. 5. Formation of hexanal (open symbols) and 2-octenal (closed symbols) by thermal decomposition of 2,4-decadienal: A, effect of $\mathrm{pH}$ in the formation of hexanal $(\mathrm{O})$ and 2octenal (O); B, effect of 2,4-decadienal concentration in the formation of hexanal $(\bigcirc)$ and 2-octenal ( $(\mathbf{O}$; C, time-course of 2,4-decadienal disappearance; D, time-course of hexanal formation; and E, time-course of 2-octenal formation. Reactions were heated at $200(\bigcirc, \boldsymbol{O}), 160(\triangle, \boldsymbol{\Delta})$, or $120^{\circ} \mathrm{C}(\nabla, \nabla)$ for $1 \mathrm{~h}$ in panels $\mathrm{A}$ and $\mathrm{B}$, and the indicated times in panels $\mathrm{C}$, $\mathrm{D}$, and $\mathrm{E}$. 


\section{Table 1}

Optimization of MRM transitions for detection of aldehydes

\begin{tabular}{|c|c|c|c|c|c|c|c|}
\hline Aldehyde & Monitored transition & $\mathrm{DP}$ & FP & EP & CEP & $\mathrm{CE}$ & CXP \\
\hline \multirow[t]{3}{*}{ Formaldehyde- $\mathrm{d}_{2}$} & $280.0 \rightarrow 156.1$ & 26 & 360 & 8 & 18 & 47 & 6 \\
\hline & $280.0 \rightarrow 115.2$ & 26 & 360 & 8 & 18 & 67 & 4 \\
\hline & $280.0 \rightarrow 171.1$ & 26 & 360 & 8 & 18 & 31 & 6 \\
\hline \multirow[t]{3}{*}{ Formaldehyde } & $278.1 \rightarrow 170.1$ & 26 & 370 & 10 & 18 & 35 & 6 \\
\hline & $278.1 \rightarrow 128.1$ & 26 & 370 & 10 & 18 & 71 & 4 \\
\hline & $278.1 \rightarrow 115.2$ & 26 & 370 & 10 & 18 & 77 & 4 \\
\hline \multirow[t]{3}{*}{ Propanal } & $306.2 \rightarrow 156.0$ & 26 & 370 & 10.5 & 14 & 53 & 6 \\
\hline & $306.2 \rightarrow 115.1$ & 26 & 370 & 10.5 & 14 & 71 & 4 \\
\hline & $306.2 \rightarrow 171.1$ & 26 & 370 & 10.5 & 14 & 31 & 6 \\
\hline \multirow[t]{3}{*}{ 2-Pentenal } & $332.2 \rightarrow 156.1$ & 26 & 370 & 10 & 14 & 55 & 6 \\
\hline & $332.2 \rightarrow 171.1$ & 26 & 370 & 10 & 14 & 37 & 6 \\
\hline & $332.2 \rightarrow 115.1$ & 26 & 370 & 10 & 14 & 79 & 4 \\
\hline \multirow[t]{3}{*}{ 2-Methyl-2-pentenal } & $346.2 \rightarrow 156.1$ & 21 & 370 & 6.5 & 26 & 57 & 6 \\
\hline & $346.2 \rightarrow 171.1$ & 21 & 370 & 6.5 & 26 & 39 & 8 \\
\hline & $346.2 \rightarrow 115.1$ & 21 & 370 & 6.5 & 26 & 79 & 6 \\
\hline \multirow[t]{3}{*}{ 2,4-Heptadienal } & $358.1 \rightarrow 170.1$ & 21 & 370 & 8 & 16 & 29 & 6 \\
\hline & $358.1 \rightarrow 171.1$ & 21 & 370 & 8 & 16 & 35 & 6 \\
\hline & $358.1 \rightarrow 115.2$ & 21 & 370 & 8 & 16 & 79 & 4 \\
\hline \multirow[t]{3}{*}{ Hexanal } & $348.1 \rightarrow 156.1$ & 26 & 370 & 10.5 & 16 & 61 & 6 \\
\hline & $348.1 \rightarrow 115.1$ & 26 & 370 & 10.5 & 16 & 83 & 4 \\
\hline & $348.1 \rightarrow 171.2$ & 26 & 370 & 10.5 & 16 & 41 & 6 \\
\hline \multirow[t]{3}{*}{ 2-Octenal } & $374.1 \rightarrow 156.1$ & 26 & 350 & 11.5 & 14 & 61 & 6 \\
\hline & $374.1 \rightarrow 171.1$ & 26 & 350 & 11.5 & 14 & 39 & 6 \\
\hline & $374.1 \rightarrow 115.1$ & 26 & 350 & 11.5 & 14 & 83 & 4 \\
\hline \multirow[t]{3}{*}{ 2,4-Decadienal } & $400.1 \rightarrow 170.0$ & 21 & 370 & 10.5 & 16 & 33 & 6 \\
\hline & $400.1 \rightarrow 171.1$ & 21 & 370 & 10.5 & 16 & 37 & 6 \\
\hline & $400.1 \rightarrow 95.1$ & 21 & 370 & 10.5 & 16 & 37 & 6 \\
\hline
\end{tabular}


Alkenals (4 or $\mathbf{7}$ )

Alkadienals ( $\mathbf{6}$ or $\mathbf{9}$ )
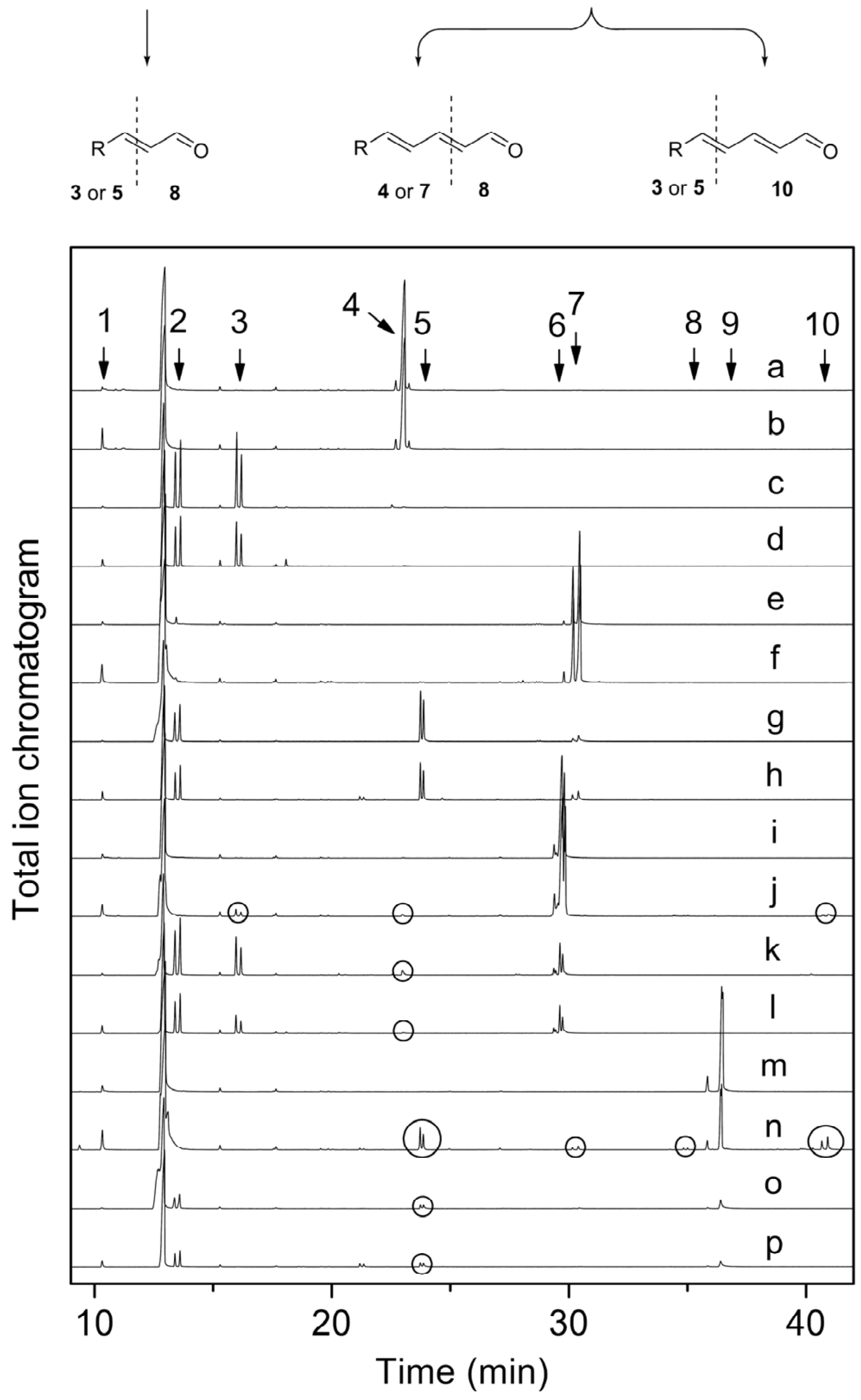

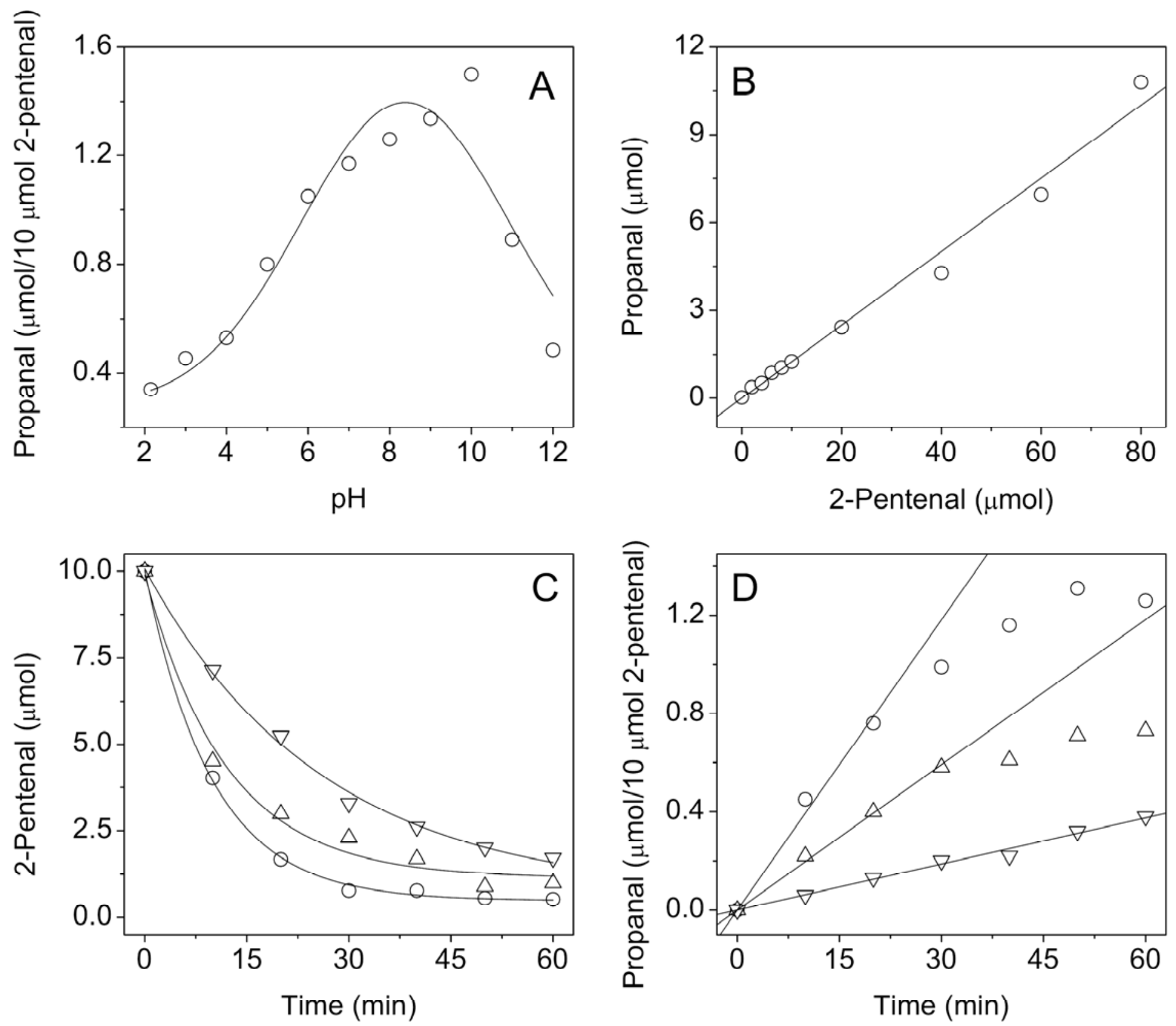

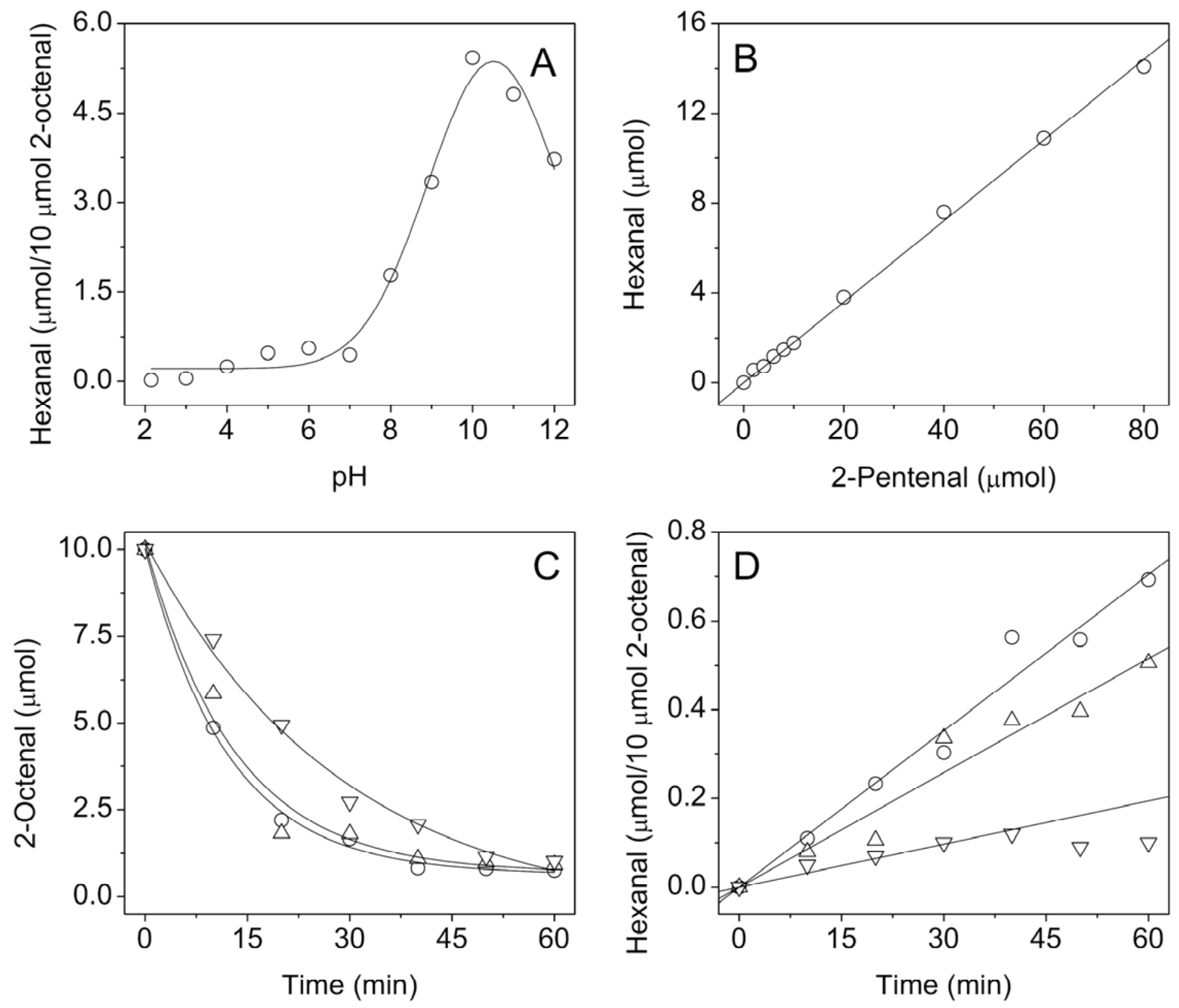

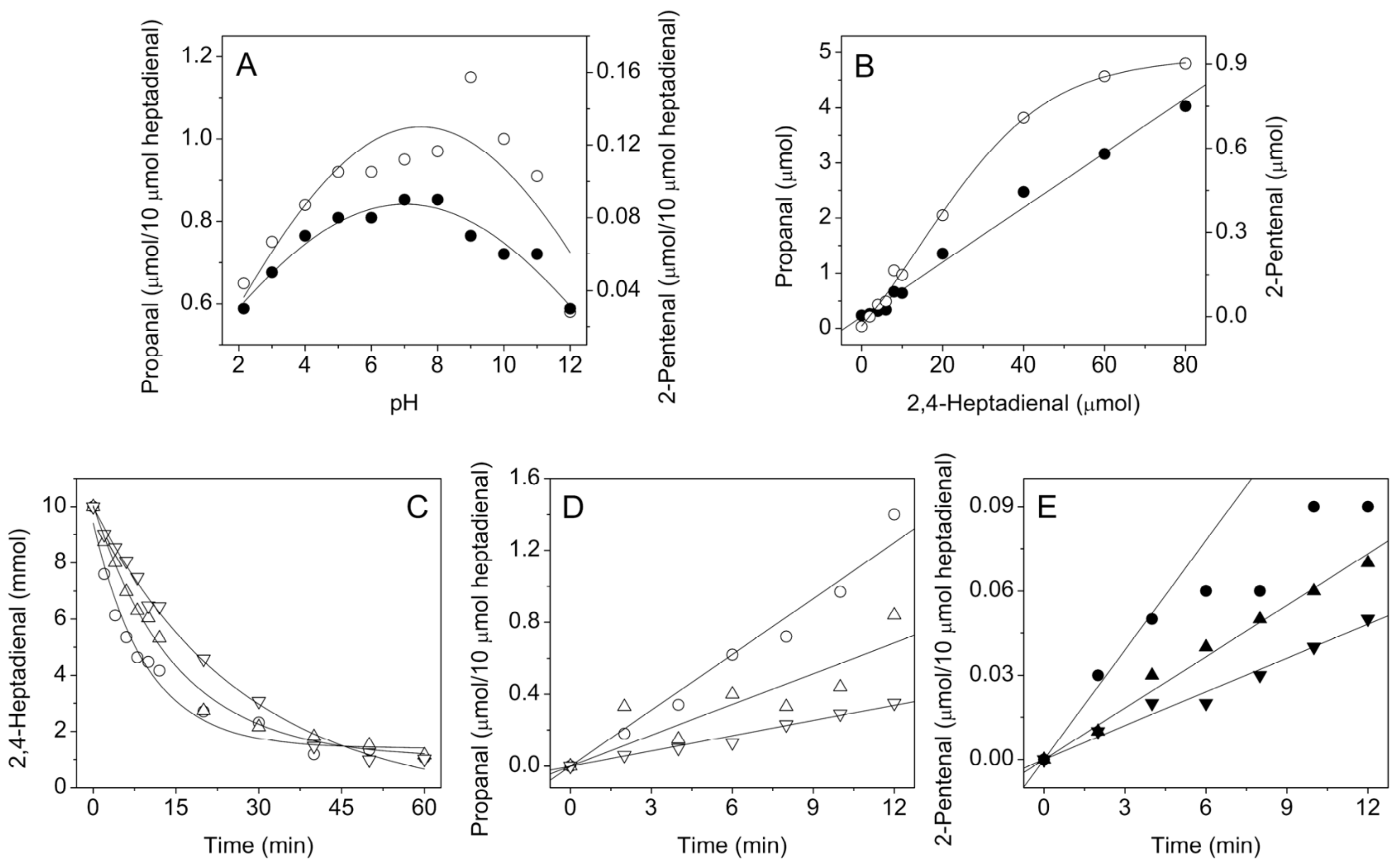

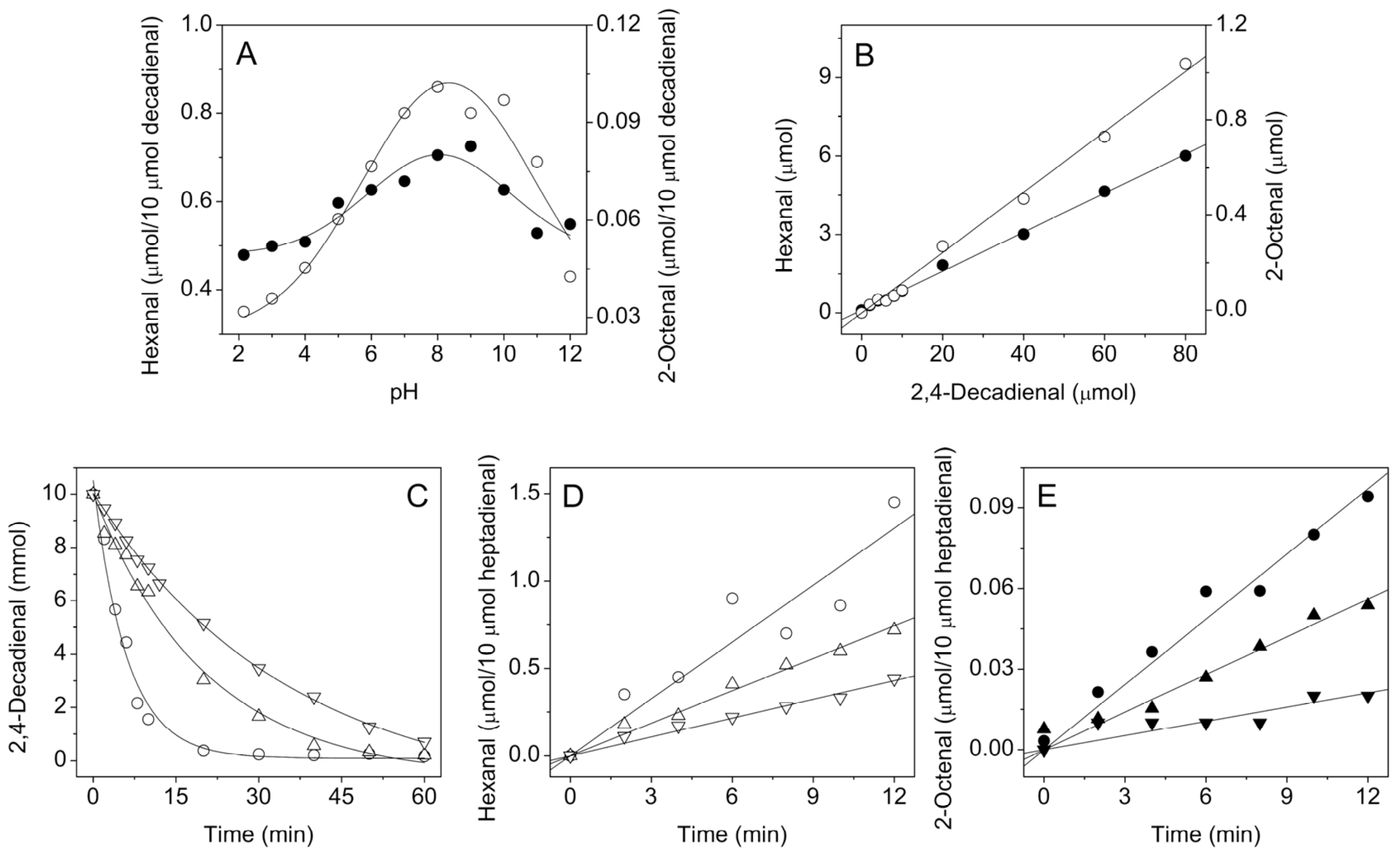